\title{
A sideway glance: a new role for endoplasmic reticulum chemical chaperones as leptin sensitizers
}

\author{
Sancia Gaetani
}

Received: 21 July 2009/Accepted: 1 August 2009/Published online: 2 September 2009

(C) Springer-Verlag 2009

The epidemic surge in the prevalence of obesity is one of the most serious public health problem confronting the western societies. Among the adult population in the USA, $65 \%$ is overweight and more than $30 \%$ is obese and the picture in Europe is becoming very similar. There has also been an increase in the obese pediatric population with one out of three Americans born in the year 2000 expected to suffer from the complications of obesity like hypertension, type- 2 diabetes and arteriosclerosis later in life [1]. However, the mechanisms behind obesity are still controversial. Obesity is usually considered as merely lifestyle disease caused by overeating and/or decreased physical activity. Although these factors have obviously great influence on weight gain, results of many investigations strongly indicate that hereditary factors could be of equal importance for the development of obesity and of its complications. Studies conducted on twins and on adoptive children show that most familial aggregation of obesity is due to genetic influence rather than to family environment. Interestingly, even in obese subjects, the weight gain throughout life is very small compared to the amount of energy ingested; and a weight gain of about $15 \mathrm{~kg}$, compared to about 50 million cal (18 tons of food) ingested in lifetime which means that the regulation of the fat depots would be off by only $0.02 \%$. This regulation must be extremely precise and the refined control mechanism is at least in part under genetic control. It has been proposed that secondary to overeating, the storage of additional fat will send signals to the brain informing that the body is overweight, inducing the subject to eat less and to burn more calories [2]. A major player in regulating energy intake and energy expenditure is leptin.

S. Gaetani $(\square)$

INRAN, Rome, Italy

e-mail: sancia.gaetani@gmail.com
This hormone protein was discovered as the product of the $o b$ gene. Mice $o b / o b$ were obese and had type-2 diabetes, due to the segregation of a single genetic locus containing the mutation. The gene is expressed only in fat cells, white and brown, and encodes a 167 amino acid-long protein, leptin. Leptin is secreted after its synthesis and functions as part of a lipostatic signaling pathway that modulates energy balance by central and peripheric effects. When fat cells increase in number and size, greater amounts of leptin are secreted into the circulation in order to maintain body weight and energy depots in equilibrium. Hypothalamus, with its central role in the regulation of hunger and satiety, is most probably the major target for leptin [3]. Treatment with recombinant leptin has resulted in a marked weight reduction in obese animals with $o b$ gene mutations as well as in normal mice. According to its function it was found also that mutations in the $\mathrm{Ob}$ receptor gene resulted in marked obesity in rodents.

These data gave hope for new treatment options in obesity, even though compared to rodents much less is known about the role of leptin in humans. Except for rare leptin-deficient individuals, obese humans are minimally responsive to leptin due to the development of leptin resistance in the brain and to defects in transportation of leptin across the blood-brain barrier. The molecular mechanisms of leptin resistance in humans are still poorly understood. Suppressor of cytokine signaling 3 (SOCS3) and tyrosine phosphatase $1 \mathrm{~B}$ (PTB1B) has been shown to play important roles in the blockade of leptin signaling [4]. In addition, recent evidence has demonstrated that increased serine phosphorylation of Janus kinase 2 (Jak2) contributes to the blockade of leptin action [5].

An article in the January 2009 issue of "Cell Metabolism" by Lale Ozcan and collaborators [6] very clearly demonstrates that increased endoplasmic reticulum (ER) 
stress and activation of the unfolded protein response (UPR) in the hypothalamus of obese mice, inhibits leptin receptor signaling.

The ER is a sophisticated luminal network in which protein synthesis, maturation, folding, and transport take place [7]. Proper ER function is critical for many aspects of cell physiology, including vesicle trafficking, lipid and membrane biogenesis and protein targeting and secretion. Metazoan cells rapidly react to ER dysfunction through a set of adaptive pathways known as ER stress response (ESR). ESR can be triggered by disparate perturbations in normal ER function, such as the accumulation of unfolded, misfolded or excessive protein, ER lipid or glycolipid imbalances, or changes in the redox or ionic conditions of the ER lumen. In response to such dysfunction, the ESR acts by both increasing the capacity of the ER to fold and process client proteins, and alleviating the burden on the organelle by reducing the amount of protein inside the ER. These effects are achieved through three major pathways: (1) the unfolded protein response (UPR), a transcriptiondependent induction of ER luminal chaperone proteins and other components of the secretory apparatus to augment the polypeptide folding and processing capacity of the ER; (2) the activation of proteasome-dependent ER-associated degradation (ERAD) to remove proteins from the ER; and (3) the control of protein translation to modulate the polypeptide traffic in the ER.

The three pathways regulate the expression of numerous genes that function within the secretory pathway, but also affect broad aspects of cell fate and the metabolism of proteins, amino acids and lipids. These pathways provide a response that remodels the secretory apparatus and aligns cellular physiology to the demands imposed by ER stress. Normally with these responses the ER homeostasis is restored. However, persistent or intense ER stress can also trigger programmed cell death or apoptosis. ER stress and apoptosis coupled to it have now been implicated in many pathologies, including obesity, diabetes, neuro-degenerative disorders, viral infection and a variety of ER storage diseases. However, in the regulation and execution steps of ER stress-induced apoptosis in mammals much remains to be understood and future research is required.

An impressive number of in vivo and in vitro experiments [6] demonstrate that obesity creates a condition of ER stress and initiates the UPR signaling pathways in the hypothalamus, which, in turn, leads to inhibition of leptin receptor signaling and creation of leptin resistance. Their observations in cellular systems rule out the possibility of a defect in leptin receptor (LepRB) folding in ER stress conditions, since translocation of LepRB to the membrane is not altered. Surprisingly, binding of leptin to LepRB increases when in the cells ER is stressed, maybe due to a decreased LepRB internalization after binding of leptin in
ER stress conditions. Moreover, acute generation of ER stress in the hypothalamus of lean mice creates a phenotype similar to that seen in the brain of obese mice. Tunicamycin infusion into the third ventricle to activate ER stress, increases the expression patterns of leptin resistance markers such as neuropeptide Y (NPY) and aguti-related peptide (AgRP) and completely blocks leptin-stimulated signal transducer and activator of transcription 3 (Stat3) activation. In a heterologous cell system, enhancement of ER capacity leads to a robust leptin-stimulated lepRB activation, suggesting that ER capacity is directly related to the leptin sensitivity. Providing additional support to this hypothesis, the authors also demonstrated that neuronal deletion of X-Box-Binding Protein 1 (XBP1), one of the master regulators of ER folding capacity, and a consequent reduction in ER function, creates severe ER stress in the hypothalamus, blocks leptin action, and generates leptin resistance in mice. The rapid and marked increase in the level of circulating leptin in the neuronal XBP1-deficient mice upon high fat feeding indicates an earlier onset of a higher level of leptin resistance.

XBP1 knockout mice (XNKO) obtained by the authors, having no obvious phenotypic abnormality, consume more food, move significantly less, gain more weight and develop significantly higher level of adiposity and obesity. While all the results provided support to the hypothesis that ER stress plays a role in the development of leptin resistance, they also raised questions of whether the hypothalamic ER stress might be reduced with chemicals and whether this could be utilized as a strategy to sensitize obese mice and humans to the anoxigenic effect of leptin.

Chemical chaperones are a group of low-molecular weight compounds that increase ER function and decrease the accumulation and aggregation of misfolded proteins in the ER lumen and consequently, reduce ER stress [8]. Two chemical chaperones are US Food and Drug Administration-approved for human utilization, 4-phenyl butyrate (PBA) and tauroursodeoxycholic acid (TUDCA). These molecules relieve ER stress in liver and adipose tissues, enhance insulin sensitivity, and maintain euglycemia in a mouse model of severe obesity and type 2 diabetes $(o b / o b)$ [9]. PBA and TUDCA also exert chaperone activity within the CNS [10]. They have been previously implicated as protective agents in the neurodegenerative diseases, where ER stress is one of the triggering mechanisms for the pathology. The authors have shown that pretreatment with PBA and TUDCA having very different chemical structure increases the leptin sensitivity of both genetic and diet-induced obesity models. Although high-dose leptin reduces the body weight of the $o b / o b$ mice, at low doses is ineffective. PBA or TUDCA coadministration with either high or low doses of leptin increases the efficacy of leptin. 
The data presented in this study suggest that overconsumption of nutrients itself could be the cause for development of leptin resistance, independently from prolonged leptin action. The results raise the possibility that increased UPR signaling even before the onset of adiposity and hyperleptinemia might be creating leptin resistance, since the leptin levels of XNKO mice severely increase at the early phases of HFD feeding, which are not proportional to the body weight.

According to the authors, the important result of the paper is the ability of both PBA and TUDCA to act as leptin sensitizers in the diet-induced obesity model. In HFD-induced obese mice, leptin administration leads to an initial reduction in body weight, but this weight loss is rapidly regained, rendering leptin an ineffective antiobesity drug. Pretreatment with the two molecules very different in chemical structure, leads to a significant weight loss in $o b / o b$ mice and also in HFD-fed diet-induced wildtype obese mice.

The results reported in the paper add new and strong data to the demonstration given in the last years by the same authors that ER stress and activation of UPR signaling pathways play a dominant role in the development of obesity-induced insulin resistance and type 2 diabetes [11] and provide important evidence that some chemical chaperones are leptin sensitizers and could represent a novel treatment option for obesity.

\section{References}

1. Muoio DM, Newgard CB (2006) Obesity related derangements in metabolic regulation. Annu Rev Biochem 76:367-401
2. Brown RE (2008) Could there be a fine-tuning role for brainderived adipokines in the regulation of bodyweight and prevention of obesity? Mcgill J Med 11:177-184

3. Gao Q, Horvath TL (2007) Neurobiology of feeding and energy expenditure. Annu Rev Neurosci 30:367-398

4. Bence KK, Delibegovic M, Xue B, Gorgun CZ, Hotamisligil GS, Noel BG, Kahn BB (2006) Neuronal PTP1B regulates body weight, adiposity and leptin action. Nat Med 12:917-924

5. Ishida-Takahashi R, Rosario F, Gong Y, Kopp K, Stancheva Z, Chen X, Feener EP, Myers MG Jr (2006) Phosphorylation of Jak2 on Ser (S23) inhibits Jak2-dependent leptin receptor signaling. Mol Cell Biol 26:4063-4073

6. Ozcan L, Ergin AS, Lu A, Chung J, Sarkar S, Nie D, Mayers MG Jr, Ozcan U (2009) Endoplasmic reticulum stress plays a central role in development of leptin resistance. Cell Metab 9:35-51

7. Ron D, Walter P (2007) Signal integration in the endoplasmic reticulum unfolded protein response. Nat Rev Mol Cell Biol 8:519-529

8. Perlmutter DH (2002) Chemical chaperones: a pharmacological strategy for disorders of protein folding and trafficking. Pediatr Res 52:832-836

9. Ozcan U, Yilmaz E, Ozcan L, Furuhashi M, Vaillancourt E, Smith RO, Gorgun CZ, Hotamisligil GS (2006) Chemical chaperones reduce ER stress and restore glucose homeostasis in a mouse model of type 2 diabetes. Science 313:1137-1140

10. Inden M, Kitamura $\mathrm{Y}$, Takeuchi $\mathrm{H}$, Yanagida $\mathrm{T}$, Takata $\mathrm{K}$, Kobayashi Y, Taniguchi T, Yoshimoto K, Kaneko M, Okuma Y, Taira T, Ariga H, Shimohama S (2007) Neurodegeneration of mouse nigrostriatal dopaminergic system induced by repeated oral administration of rotenone is prevented by 4-phenylbutyrate, a chemical chaperone. J Neurochem 101:1491-1504

11. Ozcan U, Cao Q, Yilmaz E, Lee AH, Iwakoshi NN, Ozdelen E, Tuncman G, Gorgun C, Glimcher LH, Hotamisligil GS (2004) Endoplasmic reticulum stress links obesity, insulin action, and type 2 diabetes. Science 306:457-461 